\title{
Detection of misery perfusion in the cerebral hemisphere with chronic unilateral major cerebral artery steno-occlusive disease using crossed cerebellar hypoperfusion: comparison of brain SPECT and PET imaging
}

\author{
Yoshiyasu Matsumoto $\cdot$ Kuniaki Ogasawara $\cdot$ Hideo Saito $\cdot$ Kazunori Terasaki • \\ Yoshihiro Takahashi • Yasushi Ogasawara • Masakazu Kobayashi • Kenji Yoshida • \\ Takaaki Beppu • Yoshitaka Kubo • Shunrou Fujiwara • Eiki Tsushima • Akira Ogawa
}

Received: 13 February 2013 / Accepted: 9 May 2013 / Published online: 6 June 2013

(C) The Author(s) 2013. This article is published with open access at Springerlink.com

\begin{abstract}
Purpose In patients with unilateral internal carotid or middle cerebral artery (ICA or MCA) occlusive disease, the degree of crossed cerebellar hypoperfusion that is evident within a few months after the onset of stroke may reflect cerebral metabolic rate of oxygen in the affected cerebral hemisphere relative to that in the contralateral cerebral hemisphere. The aim of the present study was to determine whether the ratio of blood flow asymmetry in the cerebellar hemisphere to blood flow asymmetry in the cerebral hemisphere on positron emission tomography (PET) and single photon emission computed tomography (SPECT) correlates with oxygen extraction fraction (OEF) asymmetry in the cerebral hemisphere on PET in patients with chronic unilateral ICA or MCA occlusive disease and whether this blood flow ratio on SPECT detects misery perfusion in the affected cerebral hemisphere in such patients.

Methods Brain blood flow and OEF were assessed using ${ }^{15} \mathrm{O}$ PET and $N$-isopropyl- $p$ - $\left[{ }^{123} \mathrm{I}\right]$ iodoamphetamine ( ${ }^{123} \mathrm{I}$-IMP) SPECT, respectively. All images were anatomically standardized using SPM2. A region of interest (ROI) was automatically
\end{abstract}

Y. Matsumoto $\cdot$ K. Ogasawara $(\bowtie) \cdot H$. Saito $\cdot$ Y. Takahashi $\cdot$

Y. Ogasawara $\cdot$ M. Kobayashi $\cdot$ K. Yoshida $\cdot$ T. Beppu $\cdot$ Y. Kubo $\cdot$

S. Fujiwara $\cdot$ A. Ogawa

Department of Neurosurgery, Iwate Medical University,

19-1 Uchimaru, Morioka 020-8505, Japan

e-mail: kuogasa@iwate-med.ac.jp

Y. Matsumoto $\cdot$ K. Ogasawara $\cdot$ H. Saito $\cdot$ K. Terasaki $\cdot$

Y. Takahashi • Y. Ogasawara $\cdot$ M. Kobayashi $\cdot$ A. Ogawa

Cyclotron Research Center, Iwate Medical University,

Morioka, Japan

E. Tsushima

Graduate School of Health Sciences, Hirosaki University,

Hirosaki, Japan placed in the bilateral MCA territories and in the bilateral cerebellar hemispheres using a three-dimensional stereotaxic ROI template, and affected-to-contralateral asymmetry in the MCA territory or contralateral-to-affected asymmetry in the cerebellar hemisphere was calculated. Sixty-three patients with reduced blood flow in the affected cerebral hemisphere on ${ }^{123} \mathrm{I}$ IMP SPECT were enrolled in this study.

Results A significant correlation was observed between MCA ROI asymmetry of PET OEF and the ratio of cerebellar hemisphere asymmetry of blood flow to MCA ROI asymmetry of blood flow on PET $(r=0.381, p=0.0019)$ or SPECT $(r=0.459$, $p=0.0001)$. The correlation coefficient was higher when reanalyzed in a subgroup of 43 patients undergoing a PET study within 3 months after the last ischemic event $(r=0.541$, $p=0.0001$ for PET; $r=0.609, p<0.0001$ for SPECT). The blood flow ratio on brain perfusion SPECT in all patients provided $100 \%$ sensitivity and $58 \%$ specificity, with $43 \%$ positive and $100 \%$ negative predictive values for detecting abnormally elevated MCA ROI asymmetry of PET OEF.

Conclusion The ratio of blood flow asymmetry in the cerebellar hemisphere to blood flow asymmetry in the cerebral hemisphere on PET and SPECT correlates with PET OEF asymmetry in the cerebral hemisphere, and this blood flow ratio on SPECT detects misery perfusion in the affected cerebral hemisphere.

Keywords Crossed cerebellar hypoperfusion · Misery perfusion · Oxygen extraction fraction - PET - SPECT

\section{Introduction}

In patients with major cerebral arterial occlusive disease, a marginally adequate blood supply relative to metabolic demand (misery perfusion) in the affected hemisphere [1] may 
increase the risk of cerebral infarction [2]. Misery perfusion can be identified by demonstrating an increased oxygen extraction fraction (OEF), which is directly measured only through positron emission tomography (PET). Several studies have identified increased OEF as a powerful and independent predictor of subsequent stroke in patients with symptomatic major cerebral arterial occlusive disease [3-5].

In clinical practice, the cerebral blood flow (CBF) response to acetazolamide can be used as an alternative parameter for detecting impaired cerebral hemodynamics [2]. Several investigators have demonstrated that cerebrovascular reactivity (CVR) to acetazolamide is inversely correlated with OEF [6-10]. Further, a subgroup of patients with symptomatic major cerebral arterial occlusive disease with reductions in both CVR to acetazolamide and CBF measured by single photon emission computed tomography (SPECT) has an increased risk of a subsequent stroke $[11,12]$. However, more than half of patients who underwent SPECT with acetazolamide challenge developed adverse effects, such as headache, nausea, dizziness, tinnitus, numbness of the extremities, and Stevens-Johnson syndrome, after administration of the acetazolamide $[13,14]$. These adverse events can have a significant effect on activities of daily living and/or ability to engage in routine employment [14].

Crossed cerebellar hypoperfusion $(\mathrm{CCH})$ is defined as a reduction in blood flow in the cerebellar hemisphere contralateral to a supratentorial lesion [15]. This phenomenon can be demonstrated on brain perfusion images obtained by SPECT or by PET [15-18]. The mechanism underlying CCH reportedly consists of disruption of the corticopontocerebellar pathway that causes functional deafferentation and transneuronal metabolic depression of the contralateral cerebellar hemisphere $[17,18]$. In patients with unilateral carotid artery occlusive disease, contralateral-to-affected side blood flow asymmetry in the cerebellar hemisphere reflects cerebral metabolic rate of oxygen $\left(\mathrm{CMRO}_{2}\right)$ in the affected cerebral hemisphere relative to that in the contralateral cerebral hemisphere [19]. Because $\mathrm{OEF}$ is a function of $\mathrm{CMRO}_{2} / \mathrm{CBF}$, the ratio of contralateral-to-affected side blood flow asymmetry in the cerebellar hemisphere to affected-to-contralateral side blood flow asymmetry in the cerebral hemisphere may reflect affected-to-contralateral side OEF asymmetry in the cerebral hemisphere. If this hypothesis is correct, then misery perfusion could be detected using only one brain blood flow imaging study without an acetazolamide challenge.

The aim of the present study was to determine whether the ratio of contralateral-to-affected side blood flow asymmetry in the cerebellar hemisphere to affected-to-contralateral side blood flow asymmetry in the cerebral hemisphere on PET and SPECT correlates with affected-to-contralateral side OEF asymmetry in the cerebral hemisphere on PET in patients with chronic unilateral middle cerebral or internal carotid artery (MCA or ICA) occlusive disease. Further, this study investigated whether this blood flow ratio on SPECT detects misery perfusion in the cerebral hemisphere ipsilateral to the affected artery in such patients.

\section{Materials and methods}

\section{Healthy volunteers}

The healthy study population consisted of 20 normal men ages 30-52 years (mean 38 years) who underwent screening based on past history, physical examination, neurological and cognitive testing, and magnetic resonance imaging (MRI) findings. Exclusion criteria included any history of hypertension, diabetes mellitus, atrial fibrillation, or pulmonary disease or observation of organic brain lesions (including leukoaraiosis or asymptomatic lacunar infarction) on MRI.

\section{Patient inclusion criteria}

Patients who satisfied the following basic inclusion criteria were prospectively selected: age $<75$ years; presence of clinical symptoms suggesting ischemic episodes in the MCA or ICA territory within 6 months before visiting our institute; absence of clinical symptoms suggesting ischemic episodes in the vertebrobasilar territory; useful residual function (modified Rankin disability scale 0,1 , or 2); presence of unilateral MCA or ICA stenosis (greater than $70 \%$ for the ICA; greater than $50 \%$ for the MCA) or occlusion on cerebral angiography using arterial catheterization, $\mathrm{CT}$, or MRI; absence of occlusion or stenosis of greater than $50 \%$ in the contralateral ICA, the contralateral MCA, the basilar artery, and the vertebral arteries; no infarct or only subcortical abnormalities (excluding infarcts involving the posterior limb of the internal capsule) in the MCA territory of the affected cerebral hemisphere on T1-, T2- and diffusionweighted MRI; absence of gross morphological alterations in the cerebellum and brain stem on T1-, T2- and diffusionweighted MRI; and provision of written informed consent. Finally, according to the brain perfusion SPECT criteria as described below (see "Brain SPECT study" and "Data analysis" sections), patients who were defined as having reduced blood flow in the affected cerebral hemisphere entered into the present study.

The study protocol was approved by the local Ethics Committee. Written informed consent was obtained from all subjects prior to enrollment in the study.

\section{Brain SPECT study}

SPECT studies were performed using a ring-type SPECT scanner, a Headtome-SET080 (Shimadzu Corp., Kyoto, Japan), which provides 31 tomographic images simultaneously [20]. 
The spatial resolution of the scanner with a low-energy, allpurpose collimator was $13 \mathrm{~mm}$ full-width at half-maximum (FWHM) at the center of the field of view, and the slice thickness was $25 \mathrm{~mm}$ FWHM at the field of view center. Image slices were taken at $5 \mathrm{~mm}$ center-to-center spacing parallel to the orbitomeatal (OM) line. The images were reconstructed using the weighted filtered backprojection technique, in which the attenuation correction was made by detecting the edge of the object. An attenuation coefficient of $0.065 \mathrm{~cm}^{-1}$, a Butterworth filter (cutoff $=0.45$ cycle $/ \mathrm{cm}$; order $=3$ ), and a ramp filter were used for image reconstruction.

The distribution of brain perfusion was assessed using $N$ isopropyl- $p$ - $\left[{ }^{123} \mathrm{I}\right]$-iodoamphetamine (IMP) and SPECT. The IMP SPECT study was performed as described previously [20]. Briefly, after a 1-min intravenous infusion of $222 \mathrm{MBq}$ of ${ }^{123}$ I-IMP (5-ml volume) at a constant rate of $5 \mathrm{ml} / \mathrm{min}$ and a 1-min infusion of physiological saline at the same rate, data acquisition was performed at a midscan time of $30 \mathrm{~min}$ for a scan duration of $20 \mathrm{~min}$. The IMP SPECT study was performed at least 3 weeks after the last ischemic attack.

\section{Brain PET study}

PET studies were performed using a SET-3000GCT/M scanner (PET/CT, Shimadzu Corp., Kyoto, Japan) [21]. This modality uses gadolinium silica oxide detectors and provides 59 slices with $2.6-\mathrm{mm}$ slice thickness. The axial field of view was $156 \mathrm{~mm}$, and the spatial resolution was $3.5 \mathrm{~mm}$ FWHM at $1 \mathrm{~cm}$ in-plane and $4.2 \mathrm{~mm}$ FWHM at the center axially. The scanner was operated in a static scan mode with dual-energy window acquisition for scatter correction. The coincidence time window was set to $10 \mathrm{~ns}$. A shield module consisting of 7-mm-thick lead plates was attached to the gantry bed and was used to cover the breast and shoulder of the subject to reduce the counting rate of random coincidence and scatter coincidence attributable to radioactivity outside the field of view.

Before the emission scan, a transmission scan (3 min) with a ${ }^{137} \mathrm{Cs}$ point source was performed with a bismuth germanate transmission detector ring coaxially attached to the gadolinium silica oxide emission detector ring. Brain blood flow was determined while the subject continuously inhaled $1,480 \mathrm{MBq}$ of $\mathrm{C}^{15} \mathrm{O}_{2}$ through a mask. Measurements of $\mathrm{CMRO}_{2}$ and $\mathrm{OEF}$ were obtained during continuous inhalation of $1,480 \mathrm{MBq}$ of ${ }^{15} \mathrm{O}_{2}$. Data were collected for $5 \mathrm{~min}$. A single breath of $444 \mathrm{MBq}$ of $\mathrm{C}^{15} \mathrm{O}$ was used to measure cerebral blood volume. Brain blood flow, $\mathrm{CMRO}_{2}$, and $\mathrm{OEF}$ were calculated using the steady-state method [22], and $\mathrm{CMRO}_{2}$ and $\mathrm{OEF}$ were corrected according to cerebral blood volume [23].

The PET study was performed between 2 and 6 days after SPECT study only for patients who were defined as having reduced blood flow in the affected cerebral hemisphere on SPECT imaging, as described below. Patients were also classified into subgroups of those undergoing a PET study within (defined as early PET study) or later than (defined as late PET study) 3 months after the last ischemic event.

Data analysis

All SPECT and PET images were transformed into the standard brain size and shape by linear and nonlinear transformation using SPM2 for anatomic standardization [24]. Thus, brain images from all subjects had the same anatomic format. Three hundred and eighteen constant regions of interest (ROIs) were automatically placed in both the cerebral and cerebellar hemispheres using a three-dimensional stereotaxic ROI template (3DSRT) with SPM2 (FUJIFILM RI Pharma Co., Ltd., Tokyo, Japan) [25]. The ROIs were grouped into ten segments (callosomarginal, pericallosal, precentral, central, parietal, angular, temporal, posterior, hippocampus, and cerebellar) in each hemisphere according to the arterial supply. Five (precentral, central, parietal, angular, and temporal) of these ten segments were combined and defined as an ROI perfused by the MCA (Fig. 1). While the MCA ROI included only the cerebral cortex, the cerebellar hemispheric ROI included the cerebellar cortex as well as the cerebellar while matter and the cerebellar ganglia. The mean value of radioactive counts on ${ }^{123}$ I-IMP SPECT images and the mean value of blood flow on PET images were measured in the bilateral MCAs and in cerebellar hemispheric ROIs; the mean value of $\mathrm{CMRO}_{2}$ and OEF on PET images was measured in the bilateral MCA ROIs. Then, the asymmetry ratio in the MCA ROI $\left(\mathrm{AR}_{\mathrm{MCA}}\right)$ was calculated as: the value in the cerebral hemisphere ipsilateral to the side of the stenosed or occluded artery/the value in the contralateral cerebral hemisphere. The asymmetry ratio in the cerebellar hemispheric $\mathrm{ROI}\left(\mathrm{AR}_{\mathrm{cbl}}\right)$ was calculated as: the value in the cerebellar hemisphere contralateral to the side of the stenosed or occluded artery/the value in the ipsilateral cerebellar hemisphere.

When $\mathrm{AR}_{\mathrm{MCA}}$ in IMP SPECT was less than 0.933, a patient was defined as having reduced blood flow in the affected cerebral hemisphere [10] and was allowed to enter into the present study.

Healthy volunteers were assigned to one of two groups, each consisting of ten subjects who underwent PET or SPECT study, respectively. In each group, the $\mathrm{AR}_{\mathrm{MCA}}$ on OEF PET or the $\mathrm{AR}_{\mathrm{cbl}} / \mathrm{AR}_{\mathrm{MCA}}$ on blood flow PET and IMP SPECT was calculated when the left cerebral hemisphere was defined as the affected side. An abnormally elevated $\mathrm{AR}_{\mathrm{MCA}}$ on $\mathrm{OEF}$ PET was defined as a value greater than the mean +2 standard deviations (SDs) obtained in the healthy volunteers.

Statistical analysis

Data are expressed as the mean $\pm \mathrm{SD}$. Correlations between various parameters were determined using linear regression 


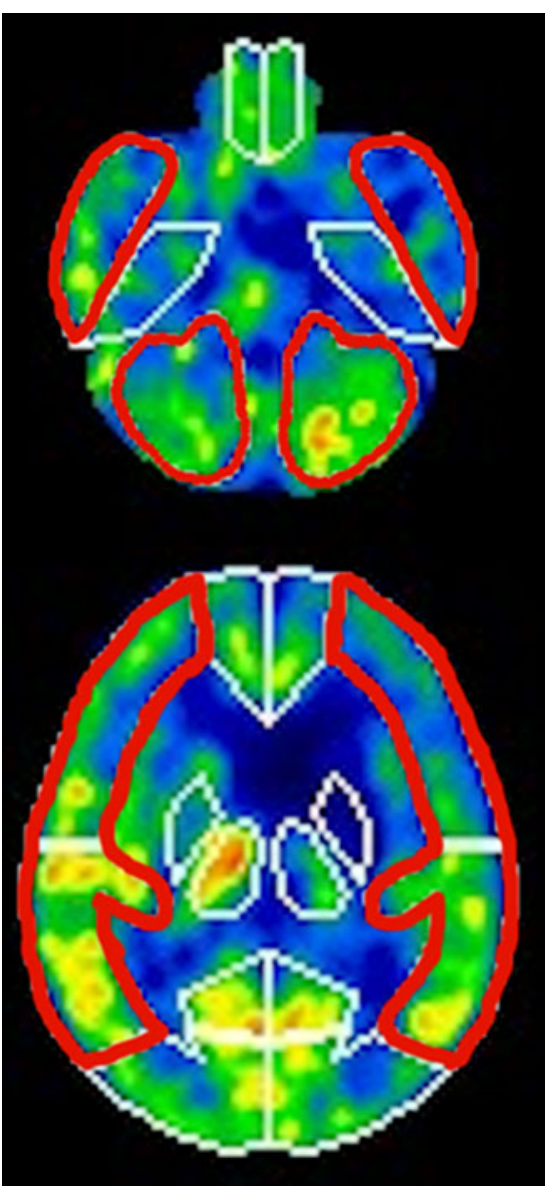

Fig. 1 Parts of the ROIs in a 3DSRT. ROIs surrounded by red lines indicate the bilateral cerebellar hemispheres and cortical territories perfused by the bilateral MCAs

analysis and by computing regression equations and correlation coefficients, and the function of better fit was determined. The analysis of covariance (ANCOVA), including Levene's test followed by pairwise comparisons, was also used to compare the intercepts of two regression lines to test the effect of the timing of PET study (early or late PET study) on a dependent variable (y-axis) while controlling for the effect of a continuous covariable (x-axis). Statistical significance was set at the $p<0.05$ level. The accuracy of $\mathrm{AR}_{\mathrm{cb} /} / \mathrm{AR}_{\mathrm{MCA}}$ on blood flow SPECT to detect abnormally elevated $\mathrm{AR}_{\mathrm{MCA}}$ on OEF PET in patients was determined by a receiver-operating characteristic curve. The curve was calculated in increments or decrements of $0.5 \mathrm{SD}$ from the mean value of $\mathrm{AR}_{\mathrm{cbl}} / \mathrm{AR}_{\mathrm{MCA}}$ on blood flow SPECT obtained in healthy volunteers.

\section{Results}

A total of 109 patients satisfied the basic inclusion criteria. Among these patients, 63 were defined as having reduced blood flow in the affected cerebral hemisphere on blood flow SPECT imaging and entered into the present study.

The mean age of the 63 patients ( 21 women and 42 men) was $59 \pm 11$ years (range $34-74$ years). Of the patients, 23 and 40 had experienced only transient ischemic attacks and minor complete strokes with or without transient ischemic attacks, respectively. Cerebral angiography demonstrated ICA stenosis in 15 patients, ICA occlusion in 27 patients, MCA stenosis in 12 patients, and MCA occlusion in 9 patients. Of the patients, 43 and 20 underwent early and late PET studies, respectively.

No significant correlation was observed between $A_{\mathrm{cbl}}$ and $\mathrm{AR}_{\mathrm{MCA}}$ on blood flow PET in patients (Fig. 2a). However, the $\mathrm{AR}_{\mathrm{cbl}}$ correlated with $\mathrm{AR}_{\mathrm{MCA}}$ on $\mathrm{CMRO}_{2}$ PET $[r=0.305$, $95 \%$ confidence interval (CI) $0.062-$ $0.514, p=0.0147]$; while the slopes of the regression lines between these two were statistically similar among equations of patients undergoing early and late PET studies, their intercepts were significantly lower in an equation of patients undergoing late PET study than in an equation of patients undergoing early PET study ( $p=0.0002$, mean difference $0.106,95 \%$ CI 0.054-0.159) (Fig. 2b). The correlation coefficient was higher when reanalyzed in the 43 patients undergoing early PET study $(r=0.522,95 \%$ CI $0.262-$ $0.711, p=0.0003$ ).

$\mathrm{AR}_{\mathrm{cbl}} / \mathrm{AR}_{\mathrm{MCA}}$ on blood flow PET correlated with $\mathrm{AR}_{\mathrm{MCA}}$ on OEF PET ( $r=0.381,95 \%$ CI $0.147-0.575, p=0.0019)$ in patients; while the slopes of the regression lines between these two were statistically similar among equations of patients undergoing early and late PET studies, their intercepts were significantly lower in an equation of patients undergoing late PET study than in an equation of patients undergoing early PET study ( $p<0.0001$, mean difference $0.164,95 \%$ CI 0.092 0.237) (Fig. 3). The correlation coefficient was higher when reanalyzed in the 43 patients undergoing early PET study $(r=0.541,95 \%$ CI $0.288-0.724, p=0.0001)$.

$\mathrm{AR}_{\mathrm{MCA}}$ on blood flow SPECT correlated with $\mathrm{AR}_{\mathrm{MCA}}$ on blood flow PET in patients ( $r=0.845,95 \%$ CI $0.755-0.903$, $p<0.0001)$; both of the slopes and intercepts of the regression lines between these two were statistically similar among equations of patients undergoing the early and late PET studies (Fig. 4a). AR $\mathrm{R}_{\mathrm{cbl}}$ on blood flow SPECT correlated with $\mathrm{AR}_{\mathrm{cbl}}$ on blood flow PET in patients $(r=0.801$, $95 \%$ CI $0.710-0.858, p<0.0001)$; both of the slopes and intercepts of the regression lines between these two parameters were statistically similar when comparing equations of patients undergoing the early and late PET studies (Fig. 4b). In half of patients, $\mathrm{AR}_{\mathrm{MCA}}$ and $\mathrm{AR}_{\mathrm{cbl}}$ were higher on blood flow SPECT than on blood flow PET. $\mathrm{AR}_{\mathrm{cbl}}$ on blood flow SPECT correlated with $\mathrm{AR}_{\mathrm{MCA}}$ on $\mathrm{CMRO}_{2}$ PET in patients $(r=0.515,95 \%$ CI $0.307-0.677, p<0.0001)$; while the slopes of the regression lines between these two were statistically similar among equations of patients undergoing 

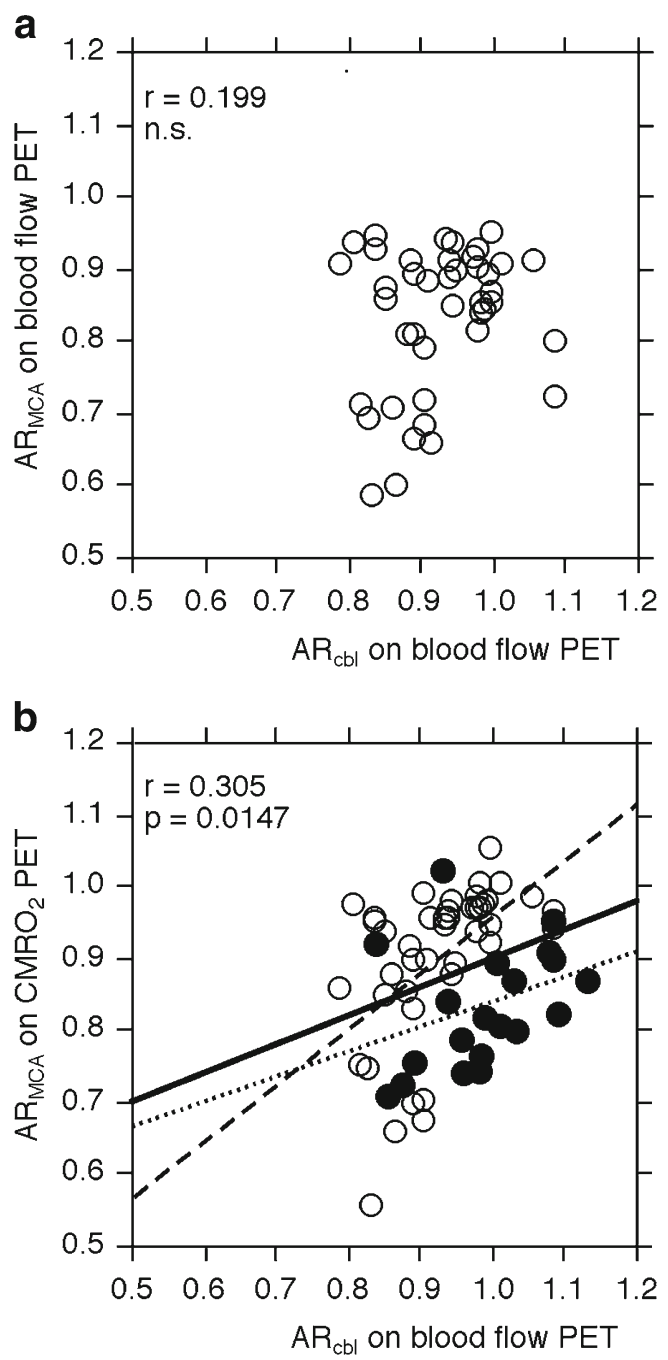

Fig. 2 Correlation between $\mathrm{AR}_{\mathrm{cbl}}$ (asymmetry ratio in the cerebellar hemispheric ROI) on blood flow PET and $\mathrm{AR}_{\mathrm{MCA}}$ (asymmetry ratio in the MCA ROI) on blood flow PET (a) or $\mathrm{AR}_{\mathrm{MCA}}$ on $\mathrm{CMRO}_{2}$ PET (b) in patients. In $\mathbf{b}$, open and closed circles denote patients undergoing early and late PET studies, respectively; solid line $(\mathrm{Y}=0.399 \mathrm{X}+0.503)$, dashed line $(\mathrm{Y}=0.783 \mathrm{X}+0.177)$, and dotted line $(\mathrm{Y}=0.346 \mathrm{X}+0.492)$ denote the regression lines of all patients, those undergoing early PET study and those undergoing late PET study, respectively

early and late PET studies, their intercepts were significantly lower in an equation of patients undergoing late PET study than in an equation of patients undergoing early PET study ( $p=0.0001$, mean difference $0.096,95 \%$ CI $0.051-0.140$ ) (Fig. 4c). The correlation coefficient was higher when reanalyzed in the 43 patients undergoing early PET study $(r=0.653,95 \%$ CI $0.439-0.797, p<0.0001)$.

$\mathrm{AR}_{\mathrm{cbl}} / \mathrm{AR}_{\mathrm{MCA}}$ on blood flow SPECT correlated with $\mathrm{AR}_{\mathrm{MCA}}$ on OEF PET $(r=0.459,95 \%$ CI $0.239-0.635$, $p=0.0001$ ) in patients; while the slopes of the regression lines between these two were statistically similar among equations of patients undergoing early and late PET studies, their intercepts were significantly lower in an equation of

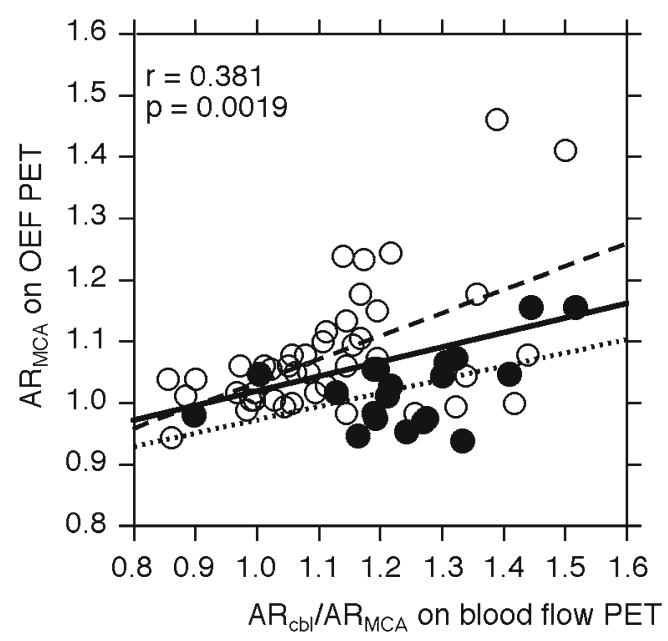

Fig. 3 Correlation between $\mathrm{AR}_{\mathrm{cb}} / \mathrm{AR}_{\mathrm{MCA}}$ on blood flow PET and $\mathrm{AR}_{\mathrm{MCA}}$ on OEF PET in patients. Open and closed circles denote patients undergoing early and late PET studies, respectively; solid line $(\mathrm{Y}=0.234 \mathrm{X}+0.792)$, dashed line $(\mathrm{Y}=0.374 \mathrm{X}+0.661)$, and dotted line $(\mathrm{Y}=0.216 \mathrm{X}+0.757)$ denote the regression lines of all patients, those undergoing early PET study and those undergoing late PET study, respectively

patients undergoing late PET study than in an equation of patients undergoing early PET study $(p=0.0032$, mean difference $0.094,95 \%$ CI $0.0328-0.155$ ) (Fig. 5). The correlation coefficient was higher when reanalyzed in 43 patients undergoing early PET study $(r=0.609,95 \%$ CI $0.363-$ $0.762, p<0.0001)$.

The $\mathrm{AR}_{\mathrm{MCA}}$ on OEF PET in healthy volunteers was $1.001 \pm 0.044$, and an abnormally elevated $\mathrm{AR}_{\mathrm{MCA}}$ on $\mathrm{OEF}$ PET was defined as a value greater than 1.089. As a result, 15 patients were classified as having an abnormally elevated $\mathrm{AR}_{\mathrm{MCA}}$ on OEF PET. The $\mathrm{AR}_{\mathrm{cbl}} / \mathrm{AR}_{\mathrm{MCA}}$ on blood flow SPECT in healthy volunteers was $0.999 \pm 0.051$, and the receiver-operating characteristic curve was calculated in increments or decrements of 0.974 from 1.484 in $\mathrm{AR}_{\mathrm{cbl}} / \mathrm{AR}_{\mathrm{MCA}}$ on blood flow SPECT. The sensitivity and specificity for the $\mathrm{AR}_{\mathrm{cbl}} / \mathrm{AR}_{\mathrm{MCA}}$ on blood flow SPECT in the cutoff point lying closest to the left upper corner of the receiver-operating characteristic curve for detection of an abnormally elevated $\mathrm{AR}_{\mathrm{MCA}}$ on OEF PET was $100 \%(15 / 15)$ and $58 \%(28 / 48)$ (cutoff point 1.101), respectively (Fig. 5). Using the same cutoff point, the positive and negative predictive values were $43 \%(15 / 35)$ and $100 \%(28 / 28)$, respectively. The cutoff point for $\mathrm{AR}_{\mathrm{cbl}} / \mathrm{AR}_{\mathrm{MCA}}$ on blood flow SPECT was the mean +2 SDs of the control value obtained from normal subjects.

When reanalyzed in 43 patients undergoing early PET study using the same cutoff point, the sensitivity, specificity, and positive and negative predictive values for the $A_{\mathrm{cbl}} / \mathrm{AR}_{\mathrm{MCA}}$ on blood flow SPECT for detection of an abnormally elevated $\mathrm{AR}_{\mathrm{MCA}}$ on OEF PET were $100 \%(13 / 13), 83 \%(25 / 30), 72 \%$ $(13 / 18)$ and $100 \%$ (25/25), respectively (Fig. 5). 

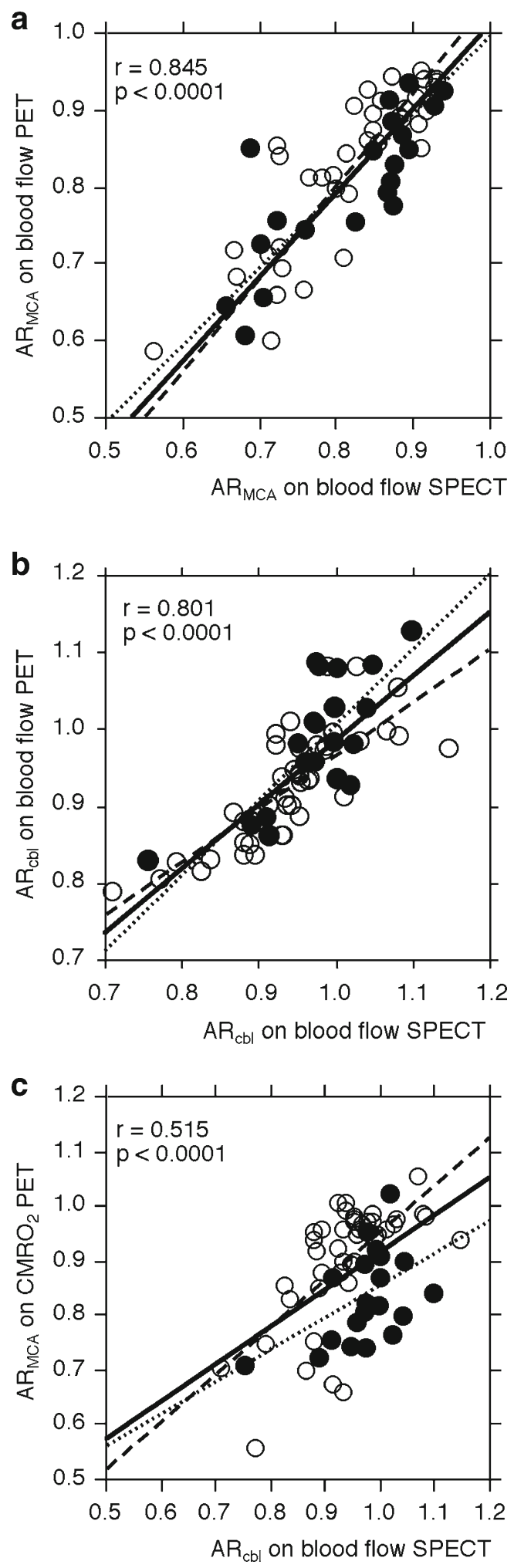

Representative PET and SPECT images in two patients with unilateral ICA occlusion and reduced blood flow in the affected cerebral hemisphere on blood flow SPECT imaging are shown in Fig. 6 (patient with an abnormally elevated
4 Fig. 4 Correlation between $\mathrm{AR}_{\mathrm{MCA}}$ on blood flow SPECT and $\mathrm{AR}_{\mathrm{MCA}}$ on blood flow PET in patients (a). Correlation between $\mathrm{AR}_{\mathrm{cbl}}$ on blood flow SPECT and $\mathrm{AR}_{\mathrm{cbl}}$ on blood flow PET in patients (b). Correlation between $\mathrm{AR}_{\mathrm{cbl}}$ on blood flow SPECT and $\mathrm{AR}_{\mathrm{MCA}}$ on $\mathrm{CMRO}_{2}$ PET in patients (c). Open and closed circles denote patients undergoing early and late PET studies, respectively; solid lines $(\mathrm{Y}=1.106 \mathrm{X}-0.087$ for $\mathbf{a}, \mathrm{Y}=$ $0.834 \mathrm{X}+0.151$ for $\mathbf{b}$, and $\mathrm{Y}=0.682 \mathrm{X}+0.232$ for $\mathbf{c})$, dashed lines $(\mathrm{Y}=$ $1.163 X+0.360$ for $\mathbf{a}, Y=0.690 X+0.275$ for $\mathbf{b}$, and $Y=0.866 X+0.090$ for $\mathbf{c})$, and dotted lines $(\mathrm{Y}=1.008 \mathrm{X}-0.012$ for $\mathbf{a}, \mathrm{Y}=0.962 \mathrm{X}+0.048$ for $\mathbf{b}$, and $\mathrm{Y}=0.591 \mathrm{X}+0.259$ for $\mathbf{c}$ ) denote the regression lines of all patients, those undergoing early PET study and those undergoing late PET study, respectively

$\mathrm{AR}_{\mathrm{MCA}}$ on OEF PET) and Fig. 7 (patient without an abnormally elevated $\mathrm{AR}_{\mathrm{MCA}}$ on OEF PET).

\section{Discussion}

The present study demonstrated that the ratio of contralateral-to-affected side blood flow asymmetry in the cerebellar hemisphere to affected-to-contralateral side blood flow asymmetry in the cerebral hemisphere on PET and SPECT correlates with affected-to-contralateral side OEF asymmetry in the cerebral hemisphere on PET in patients with chronic unilateral MCA or ICA occlusive disease.

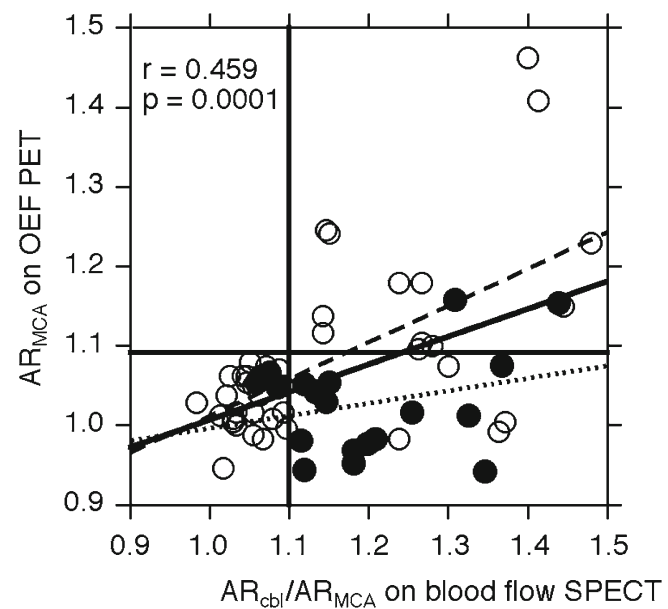

Fig. 5 Correlation between $\mathrm{AR}_{\mathrm{cbl}} / \mathrm{AR}_{\mathrm{MCA}}$ on blood flow SPECT and $\mathrm{AR}_{\mathrm{MCA}}$ on OEF PET in patients. The plot of the relationship between these two values reveals four groups of results: right upper those with elevated $\mathrm{AR}_{\mathrm{cbl}} / \mathrm{AR}_{\mathrm{MCA}}$ on blood flow SPECT and elevated $\mathrm{AR}_{\mathrm{MCA}}$ on OEF PET (true-positive); left upper those with only elevated $\mathrm{AR}_{\mathrm{MCA}}$ on OEF PET (false-negative); left lower those without elevated $\mathrm{AR}_{\mathrm{cbl}} /$ $\mathrm{AR}_{\mathrm{MCA}}$ on blood flow SPECT and without elevated $\mathrm{AR}_{\mathrm{MCA}}$ on OEF PET (true-negative); and right lower those with only elevated $\mathrm{AR}_{\mathrm{cb}} /$ $\mathrm{AR}_{\mathrm{MCA}}$ on blood flow SPECT (false-positive). Vertical and horizontal lines denote mean $+2 \mathrm{SDs}$ for $\mathrm{AR}_{\mathrm{cb}} / \mathrm{AR}_{\mathrm{MCA}}$ on blood flow SPECT and mean +2 SDs for $\mathrm{AR}_{\mathrm{MCA}}$ on OEF PET obtained in healthy volunteers, respectively. Open and closed circles denote patients undergoing early and late PET studies, respectively; solid line $(\mathrm{Y}=0.345 \mathrm{X}+0.661)$, dashed line $(\mathrm{Y}=0.467 \mathrm{X}+0.546)$, and dotted line $(\mathrm{Y}=0.157 \mathrm{X}+0.837)$ denote the regression lines of all patients, those undergoing early PET study and those undergoing late PET study, respectively 


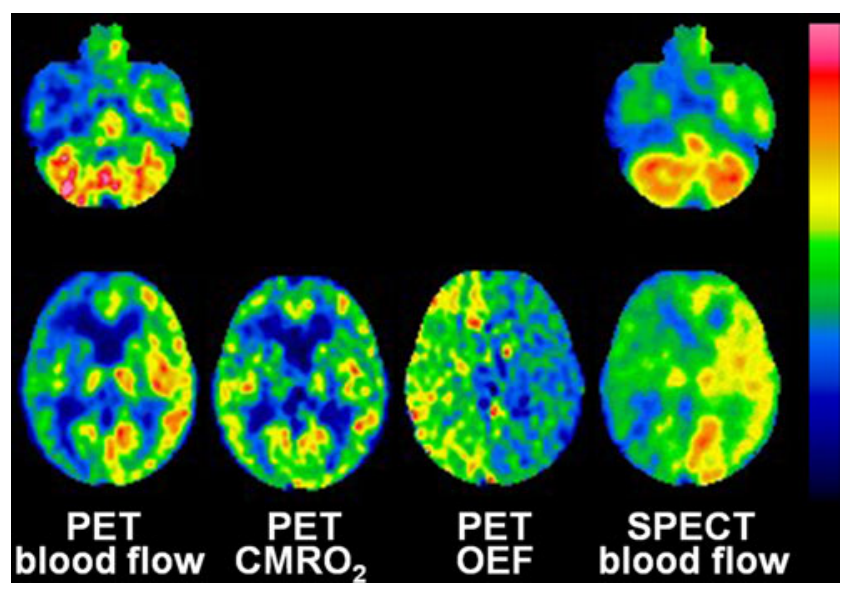

Fig. 6 A 52-year-old man with symptomatic occlusion of the right ICA. The degree of blood flow asymmetry on PET is considerably greater in the MCA territory than in the cerebellar hemisphere. $\mathrm{CMRO}_{2}$ asymmetry in the MCA territory is not observed and OEF asymmetry in the MCA territory is evident on PET. While blood flow asymmetry in the MCA territory is evident on SPECT, blood flow asymmetry in the cerebellar hemisphere is not observed on SPECT

Further, this blood flow ratio on SPECT detects misery perfusion in the affected cerebral hemisphere in such patients.

When a patient with a unilateral chronic ICA or MCA occlusive disease exhibits an increase in OEF in the ipsilateral cerebral hemisphere, blood flow in that hemisphere is essentially reduced. On the other hand, even in patients with unilateral MCA occlusive disease, the blood flow and OEF values may be decreased and increased, respectively, in the territory of the contralateral MCA as well as in the territory of the ipsilateral MCA [26]. However, in such patients, blood flow and OEF was considerably lower and higher, respectively, in the affected MCA territory than in the

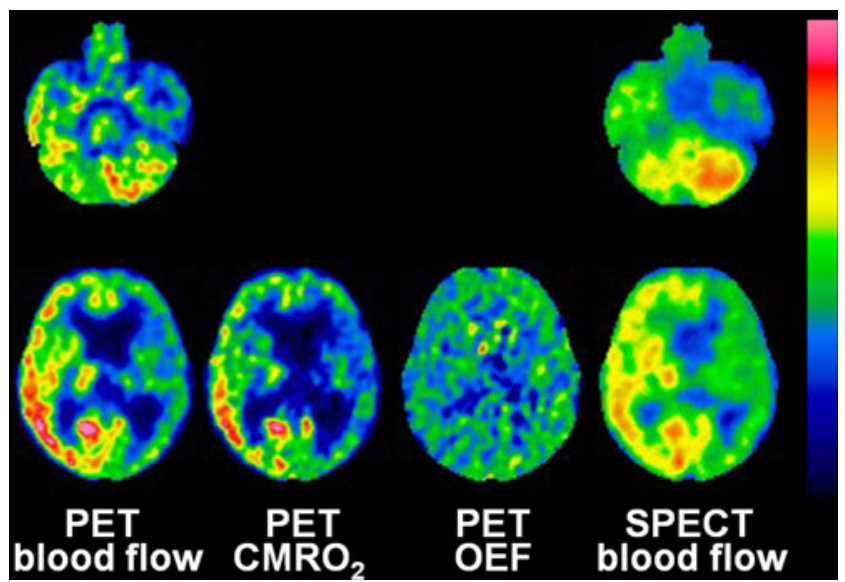

Fig. 7 A 65-year-old man with symptomatic occlusion of the left MCA. Blood flow asymmetry on PET is evident in the cerebellar hemisphere as well as in the MCA territory. $\mathrm{CMRO}_{2}$ asymmetry in the MCA territory is also evident and OEF asymmetry in the MCA territory is not observed on PET. Blood flow asymmetry on SPECT is evident in the cerebellar hemisphere as well as in the MCA territory contralateral MCA territory, and the $\mathrm{AR}_{\mathrm{MCA}}$ on brain perfusion SPECT with ${ }^{123}$ I-IMP was less than 0.933 [10]. Thus, only patients with this condition on brain perfusion SPECT with ${ }^{123}$ I-IMP finally entered into the present study.

Although the degree of blood flow asymmetry in the MCA and cerebellar hemispheric ROIs strongly correlated when comparing assessments by SPECT and PET, the degree of blood flow asymmetry was underestimated on SPECT when compared with PET in half of our patients. These findings were also illustrated in blood flow asymmetry in the cerebellar hemispheres (Fig. 6). While a previous study demonstrated a strong correlation between $\mathrm{CBF}$ as assessed by ${ }^{123}$ I-IMP SPECT and CBF as assessed by PET, the former value tended to be underestimated as CBF increased [20]. This may be mainly due to the limited firstpass extraction fraction of ${ }^{123}$ I-IMP in the brain [20].

Yamauchi et al. measured brain blood flow and metabolism using PET in patients with unilateral ICA or MCA occlusive disorders and showed that while the degree of blood flow asymmetry in the MCA territory did not differ between patients with and without $\mathrm{CCH}$, the degree of $\mathrm{CMRO}_{2}$ asymmetry in the MCA territory was significantly greater in patients with $\mathrm{CCH}$ and correlated with the degree of CCH [19]. Results from our PET studies were consistent with these previous findings and further demonstrated that the degree of $\mathrm{CCH}$ underestimated the degree of $\mathrm{CMRO}_{2}$ asymmetry in the MCA territory in patients when assessed at later than 3 months after the last ischemic event. Several studies reported that $\mathrm{CCH}$ was not always seen in patients with decreased $\mathrm{CMRO}_{2}$ in the cerebral hemisphere ipsilateral to a major cerebral artery occlusion [27] and often disappeared 2-3 months after onset in patients with hypertensive putaminal hemorrhage [28]. The present results support these findings and suggest that while $\mathrm{CCH}$ essentially reflects $\mathrm{CMRO}_{2}$ asymmetry in the cerebral hemisphere, this phenomenon may resolve over a few months after the last supratentorial cerebrovascular event despite the presence of the $\mathrm{CMRO}_{2}$ asymmetry.

Several investigators have suggested that patients with reduced $\mathrm{CBF}$ in the cerebral hemisphere ipsilateral to a major cerebral artery occlusive disease might have increased OEF in the hemisphere when $\mathrm{CCH}$ was not evident on PET [2, 19, 29]. This notion is supported by results from the present study in which the ratio of the degree of $\mathrm{CCH}$ to the degree of blood flow asymmetry in the MCA territory correlated with OEF asymmetry in the MCA territory. However, this blood flow ratio overestimated the OEF asymmetry in patients who were assessed at later than 3 months after the last ischemic event, which is related to the fact that $\mathrm{CCH}$ resolved over 3 months after the last cerebrovascular event despite the presence of $\mathrm{CMRO}_{2}$ asymmetry in the cerebral hemisphere.

Comparison of the present SPECT and PET studies demonstrated that the degree of $\mathrm{CCH}$ on SPECT correlated with 
the degree of $\mathrm{CMRO}_{2}$ asymmetry in the MCA territory on $\mathrm{PET}$ and that the ratio of the degree of $\mathrm{CCH}$ to the degree of blood flow asymmetry in the MCA territory on SPECT reflected OEF asymmetry in the MCA territory. Further, this blood flow ratio on SPECT detected misery perfusion in the affected cerebral hemisphere with a sensitivity and negative predictive value of $100 \%$. Although the specificity and positive predictive value were relatively low due to $\mathrm{CCH}$ resolving over 3 months after the last cerebrovascular event despite the presence of $\mathrm{CMRO}_{2}$ asymmetry in the cerebral hemisphere, the simplicity of requiring only the evaluation of asymmetry on blood flow images makes the present SPECT method practical. The present SPECT method also has the advantage of not requiring acetazolamide, which is otherwise associated with a significant risk of various adverse side effects $[13,14]$. In particular, this SPECT method is useful as a screening test to determine whether a PET study is needed in patients within 3 months after the last ischemic event.

The present study possesses limitations that require discussion. First, in patients with subcortical infarctions, in addition to primary ischemic cortical damage, secondary cerebral cortical hypometabolism due to a transneuronal mechanism and cerebrocerebellar tract damage are responsible for $\mathrm{CCH}$ [19]. In particular, deep infarcts destroying most of the internal capsule frequently cause severe CCH [18]. Even an infarction limited to the posterior limb of the internal capsule causes $\mathrm{CCH}$ without hypometabolism in the ipsilateral cerebral cortex [30], resulting in $\mathrm{CCH}$ without regard to cerebral cortical metabolic status in patients with unilateral ICA or MCA steno-occlusive disorders [19]. Patients with subcortical infarctions involving the posterior limb of the internal capsule were thus excluded from the present study. Second, normal values were obtained from healthy subjects who were younger than the patients who enrolled in this study. Abnormally elevated $\mathrm{AR}_{\mathrm{MCA}}$ on OEF PET was defined as a value greater than the mean +2 SDs (1.089) of the normal values. Grubb et al. categorized patients with $\mathrm{AR}_{\mathrm{MCA}}$ on OEF PET of greater than 1.082 due to unilateral ICA steno-occlusive disease as having misery perfusion and reported that such patients are at high risk for subsequent stroke when treated medically [4]. Although the cutoff point for the $\mathrm{AR}_{\mathrm{MCA}}$ on OEF PET in the present study was approximately identical to that defined by Grubb et al., whether or not the $\mathrm{AR}_{\mathrm{cbl}} / \mathrm{AR}_{\mathrm{MCA}}$ on blood flow SPECT can predict a higher risk of stroke recurrence remains unknown.

\section{Conclusion}

The ratio of contralateral-to-affected side blood flow asymmetry in the cerebellar hemisphere to affected-to-contralateral side blood flow asymmetry in the cerebral hemisphere on PET and SPECT correlates with affected-to-contralateral side OEF asymmetry in the cerebral hemisphere on PET in patients with chronic unilateral MCA or ICA occlusive disease. Further, this blood flow ratio on brain perfusion SPECT, which can be obtained using a single brain blood flow imaging study without acetazolamide challenge, detects misery perfusion in the affected cerebral hemisphere in such patients.

\section{Conflicts of interest None.}

Open Access This article is distributed under the terms of the Creative Commons Attribution License which permits any use, distribution, and reproduction in any medium, provided the original author(s) and the source are credited.

\section{References}

1. Baron JC, Bousser MG, Rey A, Guillard A, Comar D, Castaigne P. Reversal of focal "misery-perfusion syndrome" by extra-intracranial arterial bypass in hemodynamic cerebral ischemia. A case study with 15 O positron emission tomography. Stroke 1981;12:454-9.

2. Powers WJ. Cerebral hemodynamics in ischemic cerebrovascular disease. Ann Neurol 1991;29:231-40.

3. Yamauchi H, Fukuyama H, Nagahama Y, Nabatame H, Ueno M, Nishizawa $S$, et al. Significance of increased oxygen extraction fraction in five-year prognosis of major cerebral arterial occlusive diseases. J Nucl Med 1999;40:1992-8.

4. Grubb Jr RL, Derdeyn CP, Fritsch SM, Carpenter DA, Yundt KD, Videen TO, et al. Importance of hemodynamic factors in the prognosis of symptomatic carotid occlusion. JAMA 1998;280:1055-60.

5. Yamauchi H, Higashi T, Kagawa S, Nishii R, Kudo T, Sugimoto K, et al. Is misery perfusion still a predictor of stroke in symptomatic major cerebral artery disease? Brain 2012;135:2515-26.

6. Imaizumi M, Kitagawa K, Hashikawa K, Oku N, Teratani T, Takasawa $\mathrm{M}$, et al. Detection of misery perfusion with split-dose 123I-iodoamphetamine single-photon emission computed tomography in patients with carotid occlusive diseases. Stroke 2002;33: 2217-23.

7. Yamauchi H, Okazawa H, Kishibe Y, Sugimoto K, Takahashi M. Oxygen extraction fraction and acetazolamide reactivity in symptomatic carotid artery disease. J Neurol Neurosurg Psychiatry 2004;75:33-7.

8. Kuroda S, Shiga T, Houkin K, Ishikawa T, Katoh C, Tamaki N, et al. Cerebral oxygen metabolism and neuronal integrity in patients with impaired vasoreactivity attributable to occlusive carotid artery disease. Stroke 2006;37:393-8.

9. Hokari M, Kuroda S, Shiga T, Nakayama N, Tamaki N, Iwasaki Y. Combination of a mean transit time measurement with an acetazolamide test increases predictive power to identify elevated oxygen extraction fraction in occlusive carotid artery diseases. J Nucl Med 2008;49:1922-7.

10. Kuroda H, Ogasawara K, Suzuki T, Chida K, Aso K, Kobayashi $\mathrm{M}$, et al. Accuracy of central benzodiazepine receptor binding potential/cerebral blood flow SPECT imaging for detecting misery perfusion in patients with unilateral major cerebral artery occlusive diseases: comparison with cerebrovascular reactivity to acetazolamide and cerebral blood flow SPECT imaging. Clin Nucl Med 2012;37:235-40.

11. Kuroda S, Houkin K, Kamiyama H, Mitsumori K, Iwasaki Y, Abe H. Long-term prognosis of medically treated patients with internal carotid or middle cerebral artery occlusion: can acetazolamide test predict it? Stroke 2001;32:2110-6. 
12. Ogasawara K, Ogawa A, Yoshimoto T. Cerebrovascular reactivity to acetazolamide and outcome in patients with symptomatic internal carotid or middle cerebral artery occlusion: a xenon-133 single-photon emission computed tomography study. Stroke 2002;33:1857-62.

13. Ogasawara K, Tomitsuka N, Kobayashi M, Komoribayashi N, Fukuda T, Saitoh H, et al. Stevens-Johnson syndrome associated with intravenous acetazolamide administration for evaluation of cerebrovascular reactivity. Case report. Neurol Med Chir (Tokyo) 2006; 46:161-3.

14. Saito H, Ogasawara K, Suzuki T, Kuroda H, Kobayashi M, Yoshida $\mathrm{K}$, et al. Adverse effects of intravenous acetazolamide administration for evaluation of cerebrovascular reactivity using brain perfusion single-photon emission computed tomography in patients with major cerebral artery steno-occlusive diseases. Neurol Med Chir (Tokyo) 2011;51:479-83.

15. Komaba Y, Mishina M, Utsumi K, Katayama Y, Kobayashi S, Mori O. Crossed cerebellar diaschisis in patients with cortical infarction: logistic regression analysis to control for confounding effects. Stroke 2004;35:472-6.

16. Takasawa M, Watanabe M, Yamamoto S, Hoshi T, Sasaki T, Hashikawa K, et al. Prognostic value of subacute crossed cerebellar diaschisis: single-photon emission CT study in patients with middle cerebral artery territory infarct. AJNR Am J Neuroradiol 2002;23:189-93.

17. Baron JC, Bousser MG, Comar D, Soussaline F, Castaigne P. "Crossed cerebellar diaschisis": a remote functional suppression secondary to supratentorial infarction in man. J Cereb Blood Flow Metab 1981;1:s500.

18. Pantano P, Baron JC, Samson Y, Bousser MG, Derouesne C, Comar D. Crossed cerebellar diaschisis: further studies. Brain 1986;109: 677-94.

19. Yamauchi H, Fukuyama H, Yamaguchi S, Doi T, Ogawa M, Ouchi $\mathrm{Y}$, et al. Crossed cerebellar hypoperfusion in unilateral major cerebral artery occlusive disorders. J Nucl Med 1992;33:1637-41.

20. Ogasawara K, Ito H, Sasoh M, Okuguchi T, Kobayashi M, Yukawa $\mathrm{H}$, et al. Quantitative measurement of regional cerebrovascular reactivity to acetazolamide using 123I-N-isopropyl-p-iodoamphetamine autoradiography with SPECT: validation study using H2 $15 \mathrm{O}$ with PET. J Nucl Med 2003;44:520-5.

21. Ibaraki M, Miura S, Shimosegawa E, Sugawara S, Mizuta T, Ishikawa A, et al. Quantification of cerebral blood flow and oxygen metabolism with 3-dimensional PET and 15O: validation by comparison with 2-dimensional PET. J Nucl Med 2008;49:50-9.

22. Frackowiak RS, Lenzi GL, Jones T, Heather JD. Quantitative measurement of regional cerebral blood flow and oxygen metabolism in man using $15 \mathrm{O}$ and positron emission tomography: theory, procedure, and normal values. J Comput Assist Tomogr 1980;4:727-36.

23. Lammertsma AA, Jones T. Correction for the presence of intravascular oxygen-15 in the steady-state technique for measuring regional oxygen extraction ratio in the brain: 1 . Description of the method. J Cereb Blood Flow Metab 1983;3:416-24.

24. Nishimiya M, Matsuda H, Imabayashi E, Kuji I, Sato N. Comparison of SPM and NEUROSTAT in voxelwise statistical analysis of brain SPECT and MRI at the early stage of Alzheimer's disease. Ann Nucl Med 2008;22:921-7.

25. Takeuchi R, Matsuda H, Yoshioka K, Yonekura Y. Cerebral blood flow SPET in transient global amnesia with automated ROI analysis by 3DSRT. Eur J Nucl Med Mol Imaging 2004;31:578-89.

26. Tanaka M, Shimosegawa E, Kajimoto K, Kimura Y, Kato H, Oku $\mathrm{N}$, et al. Chronic middle cerebral artery occlusion: a hemodynamic and metabolic study with positron-emission tomography. AJNR Am J Neuroradiol 2008;29:1841-6.

27. Martin WR, Raichle ME. Cerebellar blood flow and metabolism in cerebral hemisphere infarction. Ann Neurol 1983;14:168-76.

28. Ishikawa Y, Mukawa J, Kinjo T, Mekaru S, Miyazato H, Takara E, et al. Crossed cerebellar diaschisis in putaminal hemorrhage - evaluation by the Xe-133 clearance method. No To Shinkei 1994;46:335-40.

29. Gibbs JM, Wise RJ, Leenders KL, Jones T. Evaluation of cerebral perfusion reserve in patients with carotid-artery occlusion. Lancet 1984;1:310-4.

30. Pappata S, Mazoyer B, Tran Dinh S, Cambon H, Levasseur M, Baron JC. Effects of capsular or thalamic stroke on metabolism in the cortex and cerebellum: a positron tomography study. Stroke 1990;21:519-24. 